\title{
Design of the Three-Dimensional Positioning Algorithm Based on RFID
}

$$
\text { Xiaohui Wang }{ }^{1,2, a^{*}} \text { and Lei Tian }{ }^{3, b}
$$

${ }^{1}$ College of Tourism and Environment, Shaanxi Normal University, Xi'an 710119, China

'Leisure Management College, Xi'an Eurasia University, Xi'an 710065, China

${ }^{3}$ School of Electronic Engineering, Xi'an University of Posts and Telecommunications, Xi'an 710121, China

âwx2324@126.con, btianlei@xupt.edu.cn

Keywords: LANDMARC; Triangulation location; Correction factor; Algorithm

\begin{abstract}
In order to realize the high precision positioning in the 3D (three dimensional) status of the LANDMARC system, using the RFID (Radio Frequency Identification) location algorithm, put forward a 3D correction algorithm with the correction factor. At the same time, applied the 3D correction algorithm in the three-dimensional space with the triangulation technology to improve the positioning accuracy. The Matlab simulation results show that the proposed method is applied to the spatial location and the calculation error is reduced by $50 \%$, which improves the reliability of the system. The technology overcomes the high cost and more reference index of the LANDMARC system high hardware.
\end{abstract}

\section{Introduction}

The research of the wireless location technology improves the rapid development of the mobile positioning technology. If the indoor positioning signal unlock, the ambiguity count of the whole week will stop [1, 2]. For the limitation of the positioning technology, the RFID technology with the characteristics of non-contact was widely applied in indoor positioning [3-5]. At present, all kinds of RFID localization algorithm are two-dimensional algorithm [6-8], this paper proposes the RFID three-dimensional correction localization algorithm, compared with the original LANDMARC system, positioning accuracy are improved significantly.

\section{RFID Location Technology}

RFID is a non-contact, automatic identification technology. Using the features of the RF (Radio Frequency) signal and completing the process of the recognition of specific objectives. At the same time, it could get the useful information from the identified goals. Because of the characteristic of the RF technology, such as the long working distance and out eyesight. It can work in all kinds of environment. The system frame structure as shown in Fig. 1:

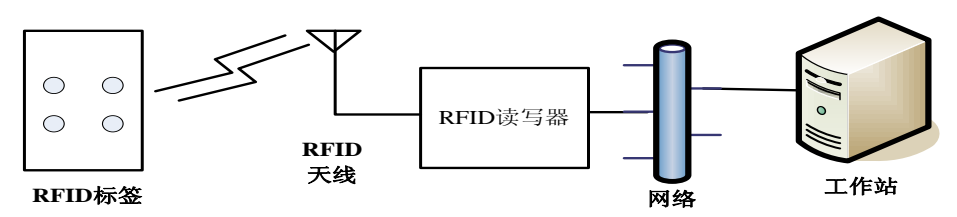

Figure 1. The RFID system structure

The system structure is mainly composed of RFID tags, read/write device and the antenna. The specific work processing is the function of the RFID antenna transmitting a certain frequency RF signal between the RFID tags and to read/write device. When the RFID tags within the scope of $\mathrm{read} / \mathrm{write}$ device, the tag was activated. At the same time, it sends the RF signals and transmits the signal through the antenna to the read/write device. RFID technology is mainly used in SpotON, 3D-iD and the LANDMARC system [9]. 


\section{LANDMARC Localization Algorithm}

The basic principle of the LANDMARC localization algorithm is the combination of the neighbor algorithm and the indirect measurement algorithm. So the main steps of the algorithm are as follows [11-13]:

1) Calculates the signal intensity of the reference tag and the locating tag to the read/write device respectively. It can be expression in Eq1.

$$
S=\sum_{i=1}^{n} a_{i} \times d^{2}
$$

Here, $\mathrm{S}$ is the signal intensity, $\mathrm{d}$ is the distance from the label to the device, $\mathrm{a}_{\mathrm{i}}$ indicates the curve fitting parameter. Because this model did not research the physical properties between the signal intensity and the responses distance. In order to get the better fitting effect, the calculation process need huge amounts of test values and the polynomial order.

2) Euclidean distance between the be-located tag $i$ and the reference tag h: If the signal intensity of $\mathrm{i}$ can be expressed as matrix $\mathrm{S}=\left[\mathrm{S}_{\mathrm{ij}}\right](\mathrm{i}=1,2 \ldots \mathrm{L} ; \mathrm{j}=1,2 \ldots \mathrm{M}), \mathrm{S}_{\mathrm{ij}}$ indicates the signal intensity from the $\mathrm{read} / \mathrm{write}$ to $\mathrm{i}$. If the signal intensity matrix of $\mathrm{h}$ is $\theta=\left[\theta_{\mathrm{ij}}\right](\mathrm{h}=1,2 \ldots \mathrm{N} ; \mathrm{j}=1,2 \ldots \mathrm{M}), \theta_{\mathrm{ij}}$ is the signal intensity from the read/write to $h$. According to the above analysis, the signal intensity Euclidean distance between $\mathrm{i}$ and $\mathrm{h}$ is $\mathrm{D}_{\mathrm{ik}}$ :

$$
D_{i h}=\sqrt{\sum_{j=1}^{M}\left(S_{i j}-\theta_{i j}\right)^{2}}
$$

The smaller the $D_{i k}$, indicates the closer between $i$ and $h$. For different value of $i$ and $h$, the $D_{i k}$ can be calculated by the same method.

3) To solve the coordinate of $i$ : First, the reference tag $\mu_{1}, \mu_{2}, \ldots \mu_{\mathrm{k}}$ which signal intensity around $\mathrm{i}$ should be mentioned, so the coordinate of i should be:

$$
\left(x_{i}, y_{i}, z_{i}\right)=\sum_{h=\mu_{1}}^{\mu_{k}} w_{i h}\left(x_{i h}, y_{i h}, z_{i h}\right)
$$

Here, $\left(\mathrm{x}_{\mathrm{ik}}, \mathrm{y}_{\mathrm{ik}}, \mathrm{z}_{\mathrm{ik}}\right)$ indicates the actual coordinate of the $\mathrm{h}$; $\mathrm{w}_{\mathrm{ik}}$ is the weight of the adjacent reference tag; so the value of $\mathrm{w}_{\mathrm{ik}}$ can be expressed:

$$
w_{i h}=\frac{1}{D_{i h}^{2}} /\left(\sum_{h=\mu_{1}}^{\mu_{k}} \frac{1}{D_{i h}^{2}}\right),\left(D_{i h} \neq 0\right)
$$

\section{D Correction Position Algorithms}

The principle of the triangulation location as showed in figure 2. A, B and C represent the three RFID read/write devices, respectively. The detect distance between the read/write device and the be-located tag are R1, R2 and R3. At this time, the three detection distance can be concluded by the signal intensity of the tag. If the coordinates of the three read/writes are know. The coordinates of the be-located tag (x', y', z') can be calculated. But there might be some error between this coordinates and the actual location coordinates $(\mathrm{x}, \mathrm{y}, \mathrm{z})$. The error $(\sigma \mathrm{x}, \sigma \mathrm{y}, \sigma \mathrm{z})$ can be expressed in Eq5.

$$
(\delta x, \delta y, \delta z)=(x, y, z)-\left(x^{\prime}, y^{\prime}, z^{\prime}\right)
$$

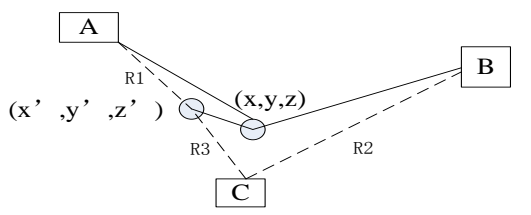

Figure 2. The principle of triangulation location 
In the normal working status, the read/write device working in the continuous mode, by the requirement of each tag can be detected by more than three read/write devices at the same time. In order to route the calculate process, assuming the number of RFID read/write devices are n, reference tag is $\mathrm{m}$, and $\mathrm{u}$ is the be-located tag. The specific calculation process is:

1) Get the number of the read/write devices $p, p \in[3, n]$ for detect the be-located tag;

2) From the step 1), choose the $q$ read/write devices in $p$, and detect the reference tag for $r$;

3) Calculates the vector $\bar{D}=\left(D_{1}, D_{2}, D_{3} \cdots D_{r}\right)$ between the be-located tag and the reference tag $\mathrm{r}$, and choose the nearest neighbors $\mathrm{k}$;

4) According to the triangulation location method, using the $q$ read/write devices calculate the location of the nearest neighbors coordinates $\left(\mathrm{x}_{\mathrm{i}}, \mathrm{y}_{\mathrm{i}}, \mathrm{z}_{\mathrm{i}}\right)$. The actual location of the keference tag is $\left(\mathrm{x}_{\mathrm{i}}, \mathrm{y}_{\mathrm{i}}, \mathrm{z}_{\mathrm{i}}\right)$. With the equation (4), the average error $\left(\sigma \mathrm{x}_{\mathrm{i}}, \sigma \mathrm{y}_{\mathrm{i}}, \sigma \mathrm{z}_{\mathrm{i}}\right)$ can be expressed $(\delta x, \delta y, \delta z)=\left(\frac{1}{k} \sum_{i=1}^{k} \delta x_{i}, \frac{1}{k} \sum_{i=1}^{k} \delta y_{i}, \frac{1}{k} \sum_{i=1}^{k} \delta z_{i}\right)$;

5) By the triangulation location principle, using the coordinates ( $\left.x^{\prime}, y^{\prime}, z^{\prime}\right)$ in the step 2) and the equivalent expression $(\mathrm{x}, \mathrm{y}, \mathrm{z})=(\mathrm{x}, \mathrm{y}, \mathrm{z})+\left(\sigma \mathrm{x}_{\mathrm{i}}, \sigma \mathrm{y}_{\mathrm{i}}, \sigma \mathrm{z}_{\mathrm{i}}\right)$ to calculate the actual coordinates $(\mathrm{x}, \mathrm{y}, \mathrm{z})$ of the be-located tag;

6) The average error of the be-located tag is $e_{i}=\sqrt{\left(x-x^{\prime}\right)^{2}+\left(y-y^{\prime}\right)^{2}+\left(z-z^{\prime}\right)^{2}}$.

\section{The Algorithm Simulation}

Combined with the 3D corrected positioning method, suppose the number of the RFID read/write device is 4, the number of the reference tag is 20 and the be-located tag is 10 . The specific simulation diagram as shown in Fig. 3:

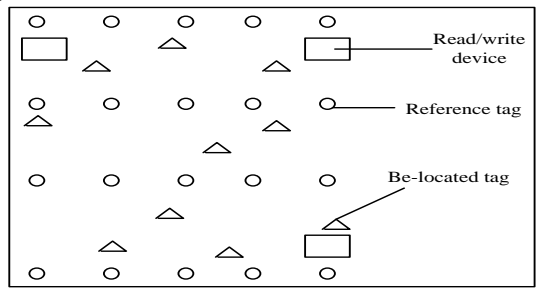

Figure 3. The diagram of the read/write device stag

According to the process in the section three and the setting parameters $\mathrm{q}=3$ and $\mathrm{k}=4$, simulated 100 times for the 10 be-located tags, the nearest coordinates of the reference tag $\left(x_{i}^{\prime}, y_{i}^{\prime}, z_{i}^{\prime}\right) i=1,2$, 3, 4 was achieved. For the coordinates of the nearest reference tag is $\left(x_{i}, y_{i}, z_{i}\right) i=1,2,3,4$.

So it can be included $\left(\delta x_{i}, \delta y_{i}, \delta z_{i}\right)=\left(x_{i}, y_{i}, z_{i}\right)-\left(x_{i}^{\prime}, y_{i}^{\prime}, z_{i}^{\prime}\right)$. The average value is $(\delta x, \delta y, \delta z)=\frac{1}{4} \sum_{i=1}^{4}\left(\delta x_{i}, \delta y_{i}, \delta z_{i}\right)$, then the coordinates of the be-located tag is $(\mathrm{x}, \mathrm{y}, \mathrm{z})=\left(\mathrm{x}^{\prime}, \mathrm{y}^{\prime}, \mathrm{z}^{\prime}\right)$ $+(\sigma \mathrm{x}, \sigma \mathrm{y}, \sigma \mathrm{z})$.

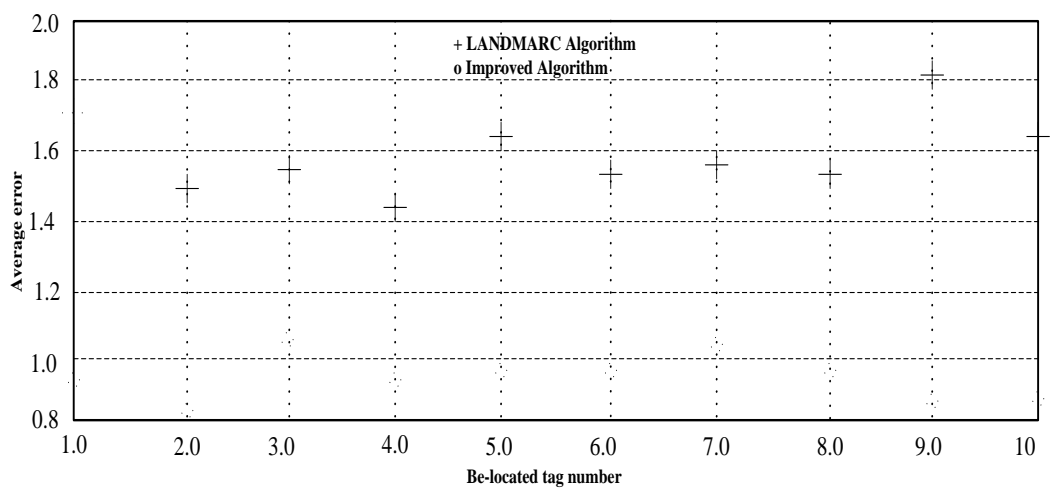

Figure 4. Comparison between the improved algorithm and the LANDMARC algorithm 
From the Fig. 4, the 3D corrected location algorithm in this paper has lower error. It drops nearly double than the original LANDMARC algorithm. Then put this 3D error corrected location algorithm into the spatial orientation, the calculation error could be reduce sharply and the system reliability would improved.

\section{Conclusion}

This paper uses the pseudo distance difference method, combining with the principle of the RFID location LANCMARC system, puts forward a three-dimensional correction localization algorithm. It corrects the positioning accuracy by the correction factor. The algorithm was used in the 3D indoor positioning. This algorithm not only reduces the location area but also improve the positioning accuracy by the correction factor.

\section{Acknowledgement}

This work was partly supported by the Shaanxi Provincial Department of education scientific research project (No. 15JK1676) of China, the project (2015EA03) supported by the key project of social science planning of Xi'an City and the Youth Founded Project of Xi'an University of Posts and Telecommunications (101-0488).

\section{References}

[1] Q.H.Zhang, W.Li. Second International Conference on Computer Modeling and Simulation (Sanya, Hainan, 2010).Vol.4, p.93-95.

[2] R.Y, Zhong, G.Q.Huang, and S, Lan. International Journal of Production Economics, Vol. 165(2015), p.260-272.

[3] P.Urien, Piramuthu, S.P.Urien. Decision Support Systems, Vol. 59 (2014), No.1, p. 28-36.

[4] E.Digiampaolo, F.Martinelli. Transactions on Industrial Electronics, Vol.61, No.1 (2014), p.365-376.

[5] E.Welbourne, L.Battle and G.Cole. IEEE Internet Computing, Vol.13, No.3 (2009), p.48-55.

[6] K. Domdouzis, B. Kumar, C. Anumba. Advanced Engineering Informatics, Vol.2007, No. 21(4) p.350-355.

[7] S.S.Saab, Z.S. Nakad. IEEE Transactions on Industrial Electronics, Vol. 58(2011), No. 5, p.1961-1970.

[8] X.Huang, Z.Z.Wang and J.H.GUO. Journal of Central South University of Technology, No.23 (2016), p.189-200.

[9] E.Digiampaolo, F.Martinelli. IEEE Transactions on Industrial Electronics, Vol.59 (2012), No. 10, p.3961-3970.

[10] Y. H.Chen, S.J.Horng and R.S. Run. IEEE Transactions on Industrial Informatics, Vol.6 (2010), No. 1, p.105-121.

[11]W.Chong, H.Y.Wu and N.F.Tzeng. 26th IEEE International Conference on Computer Communications. (Anchorage,AK,2007) Vol.2007, p.1235-1243.

[12] R.Y.Zhong, S.Lan, and C.Xu. International Journal of Advanced Manufacturing Technology, Vol.84 (2016), No. 1-4, p.1-12. 